\title{
Aktivierung zur Beteiligung im betrieblichen Arbeits- und Gesundheitsschutz
}

\author{
Karina Becker \\ Thomas Engel \\ Diana Lehmann
}

Die Aktivierungsrhetorik ist in der Betriebspolitik angekommen: Beschäftigte und ihre Interessenvertreter müssen sich mit Forderungen wie die des Lohnverzichts im Krankheitsfall auseinandersetzen - eine Selbstbeteiligung zur Stärkung von Eigenverantwortung, so die Argumentation der Arbeitgeberseite. Die Disziplinierungswirkung solcher Appelle ist nicht zu unterschätzen, wie der folgende Beitrag zeigt. Dabei ließe sich Aktivierung positiv wenden, wenn Beschäftigte gemeinsam die Verbesserung ihrer Arbeitsbedingungen und Gesundheitssituation vorantreiben. Der Beitrag zeigt anhand eines Unternehmensfalles, wie Freiräume als Voraussetzung von Mitsprache und Beteiligung im betrieblichen Arbeits- und Gesundheitsschutz genutzt werden können, aber auch erkämpft und verteidigt werden müssen.

\section{Aktivierung als Disziplinie- rungsprogramm}

In der Debatte um die Neuausrichtung der Arbeitsmarktpolitik und die Erneuerung des Sozialgesetzbuches unter der damaligen rot-grünen Regierung wurde immer auch die Stärkung individueller Eigenverantwortung als Ziel betont. Zumindest glaubte man, diese durch eine Aktivierungspolitik „fördern und fordern“ zu können. Daraus leitete sich ab, dass gesellschaftliche Teilhabe nicht ausschließlich durch Erzielung von Einkommen mit so die Kritik - Effekten von „solidarisch finanzierten Ruhezonen“ (Lessenich 2008, S. 94) zu gewährleisten sei, sondern vermittelt über die eigenverantwortliche, aktive Bemühung um Teilnahme am Erwerbsleben zu erfolgen habe (ebd., S. 88f.). In der Kurzformel „Mehr Eigenverantwortung, die zu Gemeinwohl führt" (Schröder 2000, S. 2001) klingt der verpflichtende Charakter an, demzufolge Eigenverantwortung vor allem zur Stärkung der sozialen Gemeinschaft gewünscht ist. In den 1990er Jahren hatte sich der Mainstream der politischen Parteien und vieler Verbände auf das gemeinsame gesellschaftliche Ziel „Vorfahrt für Arbeit" geeinigt. Mehr Arbeitsplätze waren demnach nur zu haben, wenn sich der Einzelne auf eine stärkere Übernahme von Verantwortung einließe.

Sowohl Fragen der Teilhabe an betrieblichen und gesellschaftlichen Entscheidungsprozessen als auch Fragen der „Qualität von Arbeit“ wurden in diesem als , ar- beitspolitisch verlorenes Jahrzehnt" gekennzeichneten Zeitraum (Pickshaus 2007; Sauer 2009) hintangestellt. Gleichwohl hielten auch im betriebspolitischen Feld Aktivierungsrhetorik und damit verbundene Verantwortungsverlagerung Einzug. „Der Aktivierungsdiskurs durchzieht praktisch alle Bereiche der Sozialpolitik" (Opielka 2008) und wird - wie auch in der Arbeitsmarktpolitik - begleitet vom Ruf nach mehr Eigenverantwortung und individuellem ökonomischen Handeln. So forderte der Präsident der Deutschen Industrieund Handelskammer (DIHK) eine Beteiligung von Beschäftigten an den krankheitsbedingten Kosten, indem „Arbeitnehmer im Krankheitsfall in den ersten beiden Tagen keine Lohnfortzahlung erhalten. Eine solche Selbstbeteiligung der Arbeitnehmer stärkt die Eigenverantwortung" (Die Welt 26.08.2005).

Vorschläge dieser Art haben auf die mikropolitischen Aushandlungen in den Betrieben Einfluss, weil sie die betrieblichen Parteien mit Argumenten und Machtressourcen versorgen. Damit lancieren sie eine Entwicklung, in der die Externalisierung von Verantwortung und Kosten von Seiten des Staates wie der Arbeitgeber - zur tonangebenden Strategie wird. Beispiele hierfür sind die Privatisierung von Zahnersatz und Krankentagegeldversicherung sowie die Einführung der Praxisgebühr im Rahmen der Gesundheitsreform 2003. Die paritätische Verteilung der Krankenkassenbeiträge auf Arbeitnehmer und Arbeitgeber - jahrzehntelang in der Bundesrepublik unantastbarer Konsens - gilt vorerst als beendet. Es wird sich zeigen, ob der als vorübergehender Aufschlag geplan- te Kostenanstieg für die Beschäftigten bald wieder zurückgenommen wird. Aktuell verstetigt die Politik z.B. durch den Gesundheitsfond jedoch den Selbstbeteiligungskanon, wie er seit der Agenda 2010 vielstimmig erklingt.

Betrieblich lässt sich dieser Wandel auch an der Weigerung vieler Unternehmen ablesen, für „verschlissene“, nicht mehr „olympiareife“ Arbeitskräfte die Verantwortung zu übernehmen. Hinter dem Appell zur Übernahme von Eigenverantwortung stecken daher auch handfeste Disziplinierungsabsichten, die, wie es der Vorschlag des Präsidenten des Deutschen Industrie- und Handelskammertags (DIHK) zeigt, zu Selbstdisziplinierung führen sollen. Darauf ausgerichtete Praktiken sind

Karina Becker, Dr., Wissenschaftliche Mitar-
beiterin im Bereich Arbeits-, Industrie- und
Wirtschaftssoziologie der Universität Jena.
Arbeitsschwerpunkte: Finanzmarktkapitalis-
mus, KMU, Gesundheit und Markt.
e-mail: karina.becker@uni-jena.de
Thomas Engel, Wissenschaftlicher Mitarbei-
ter im Bereich Arbeits-, Industrie- und Wirt-
schaftssoziologie der Universität Jena.
Arbeitsschwerpunkte: Arbeitssoziologie,
Arbeits- und Gesundheitsschutz, Industrielle
Beziehungen, demografischer Wandel.
e-mail: thomas.enge/@uni-jena.de
Diana Lehmann, Wissenschaftliche Mitarbei-
terin im Bereich Arbeits-, Industrie- und
Wirtschaftssoziologie der Universität Jena.
Arbeitsschwerpunkte: Arbeitsmarkt, Prekari-
täts-, Frauen- und Geschlechterforschung.
e-mail: diana.lehmann@uni-jena.de
beiterin im Bereich Arbeits-, Industrie- und Wirtschaftssoziologie der Universität Jena. Arbeitsschwerpunkte: Finanzmarktkapitalismus, KMU, Gesundheit und Markt. schaftssoziologie der Universität Jena. Arbeitsschwerpunkte: Arbeitssoziologie, Arbeits- und Gesundheitsschutz, Industrielle Beziehungen, demografischer Wandel. terin im Bereich Arbeits-, Industrie- und Wirtschaftssoziologie der Universität Jena. täts-, Frauen- und Geschlechterforschung. e-mail: diana.lehmann@uni-jena.de 
keine Seltenheit mehr. Aktuell fordert etwa die Münchener Flughafengesellschaft die Beschäftigten bei Verdacht auf erschlichene Krankheitstage unter Androhung der Streichung der Lohnfortzahlung dazu auf, Ärzte von ihrer Schweigepflicht zu entbinden (Süddeutsche Zeitung 10.01.2009). In Erscheinung tritt somit eine als Übernahme von Eigenverantwortung plakatierte, letztlich aber mit disziplinierender Absicht und Kostensparstrategie verknüpfte betriebliche Arbeitspolitik.

Im folgenden Beitrag wird nach den Risiken, Chancen und Voraussetzungen für eine Aktivierungspolitik im erneuerten betrieblichen Arbeits- und Gesundheitsschutz (AGS) gefragt. Die theoretischen Überlegungen und empirischen Grundlagen - Interviews mit Beschäftigten, Betriebsräten und Experten - sind im Kontext eines noch laufenden Forschungsprojektes zum betrieblichen Gesundheitsschutz entstanden. ${ }^{1}$

\section{2 \\ Der neue Arbeits- und Gesundheitsschutz}

\subsection{AKTIVIERUNGSPOSTULAT}

Es lassen sich zwei grundlegende Veränderungstendenzen im betrieblichen Arbeits- und Gesundheitsschutz identifizieren: Ausgehend von der Umsetzung einer neuen EU-Rahmenrichtlinie wurden in Deutschland, wie in vielen anderen Mitgliedstaaten, die gesetzlichen Grundlagen des betrieblichen AGS mit Veränderungen in Richtung (1) Deregulierung und (2) Modernisierung geändert.

(Ad 1) Das auf der Grundlage der EURichtlinie novellierte deutsche Arbeitsschutzrecht zielt auf einen an den betrieblichen Anforderungen orientierten, flexibilisierten und zunehmend eigenverantwortlich $\mathrm{zu}$ gestaltenden betrieblichen AGS und reagiert damit auf den Vorwurf staatlicher „Überregulierung“, wie von Unternehmerseite immer wieder geäußert wird. An die Stelle eines stark reglementierten Modells (durch technische Normen, Grenzwerte, Vorschriften) tritt damit ein auf der betrieblichen Ebene ausgehandelter Gesundheitsschutz (Reusch 2009), dessen Grenzen und Normen durch die betrieblichen Akteure selbst auszuhandeln sind.
(Ad 2) Ein zentraler Modernisierungsimpuls ergibt sich aus der gesetzlichen Forderung, Beschäftigte und ihre Interessenvertretungen systematisch in den betrieblichen AGS einzubinden. So regelt das Betriebsverfassungsgesetz ( $\$ 87$ BetrVG) auch den Anwendungsbereich des Arbeitsschutzgesetzes ( $\$ \$ 3,5$ ArbSchG) für die Arbeit von Interessenvertretungen. Das Gesetz zielt auf die systematische Beteiligung und Aufwertung von Beschäftigten praktisch als „Experten ihrer eigenen Gesundheit“. Damit werden sie nicht mehr nur als zu schützende Objekte gesehen, sondern als aktive Subjekte mit Rechten, Pflichten und Interessen. Den Beschäftigten öffnet das Gesetz damit neue Beteiligungswege und -felder. ${ }^{2}$

Der Beteiligungs- und Partizipationsanspruch des erneuerten Arbeits- und Gesundheitsschutzes als strukturelle Verantwortungsverlagerung auf die Akteursebene entspricht zunächst dem gängigen Aktivierungspostulat, so die erste These unseres Beitrages. Diese spezifische Entsprechung des Aktivierungsdiskurses intendiert, Individuen eigenverantwortlich-aktiv und damit per se gemeinwohlförderlich in Anspruch zu nehmen.

\subsection{DAS RISIKO DER BETEILIGUNG}

Im betrieblichen Alltag haben Arbeitschutz und Gesundheitsförderung bei Beschäftigten jedoch einen vergleichsweise geringen Stellenwert. Natürlich erfüllen Betriebe in der Regel formale gesetzliche Auflagen. Auch setzt sich die Erkenntnis durch, auf das Altern von Belegschaften und die Heraufsetzung des Rentenalters betriebspolitisch reagieren, zumindest vorbereitet sein zu müssen (Morschhäuser 2003). Die daran geknüpften Überlegungen und Strategien zielen jedoch eher auf eine veränderte Personalrekrutierung, im besten Fall auf eine Anpassung der Aus- und Weiterbildung, selten aber auf eine Neuausrichtung der betrieblichen Gesundheitspolitik, die demgegenüber hauptsächlich kurzfristigen Kostenerwägungen untergeordnet wird (Becker et al. 2007). Management und Belegschaft verlassen sich zumeist darauf, dass für AGS-Fragen zuständige Experten ihre Aufgaben in eigener Verantwortung wahrnehmen und bewältigen.

Mit Blick auf die betriebliche Realität bietet der neue gesetzliche Rahmen für die Beschäftigten weniger „Haltegriffe“, die das Grundrecht auf aktive Teilhabe abzusi- chern helfen. In einer Situation weitgehend fehlender konkreter Normensetzung und -durchsetzung sind Geschäftsleitungen in der Regel in einer machtvolleren Position. Ein Gesundheitsexperte der Berufsgenossenschaft bringt das Problem der Durchsetzung von Standards ohne Rechtsgrundlage wie folgt zum Ausdruck:

„Ein Beispiel: Es wird ein Mangel im Betrieb festgestellt, den ich dem Unternehmer rüberbringe. Nun kann der sagen, Ja ich mache das, ich beseitige diesen Mangel, und dann ist das erledigt.' Oder der sagt, ,Ich mache es nicht, denn wo steht geschrieben, dass ich das verändern muss? Und das ist genau der Punkt, wo man dann ins Schleudern kommt als Berufsgenossenschaft, weil es eben die konkrete Vorschrift nicht mehr gibt. Da gab es keine Diskussion. Jetzt sagt der Unternehmer: ,Ja, muss ich denn das?' Da muss ich ihm sagen: ,Sie müssen nicht, sie können aber, es wäre schön, wenn Sie würden.' Dann sagt der: ,Aber ich will nicht"“ (Gesundheitsexperte Berufsgenossenschaft).

Die gesetzliche Neuorientierung, die Kontrolle der bleibenden Normen an die Betriebe zu delegieren und somit die vormalige Kontrolle von außen durch innerbetriebliche Prävention zu ersetzen, führt in der betrieblichen Praxis vielfach zu einer Situation, in der den Beschäftigten zunehmend schlechte AGS-Standards zugemutet werden (Fuchs 2007). In diesem oftmals schleichenden Prozess werden Routinekontrollen vernachlässigt, Sicherheitsvorrichtungen nicht oder nur unzureichend überprüft und Termine für Arbeitsschutzunterweisungen verschoben. Insbesondere bei der wachsenden Gruppe der prekär Beschäftigten lässt sich beobachten, dass Gesundheit und Gesundheitsschutz zugunsten von Unternehmenszielen zurückgestellt werden und Ansprüche in diesem Bereich zurückgeschraubt oder erst gar nicht artikuliert werden. Es ist vor allem diese Beschäftigtengruppe, die eine „Selbstbewirtschaftung der eigenen Gesundheit" betreibt und damit dem Ruf der Arbeitgeber und der Politik nach Übernahme von Ei-

\footnotetext{
Das Projekt wird gefördert vom Bundesministerium für Arbeit und Soziales (BMAS) und fachlich begleitet durch die Bundesanstalt für Arbeitsschutz und Arbeitsmedizin (BAuA). Weitere Informationen unter: www.grazil.net

2 Auch das novellierte Betriebsverfassungsgesetz bietet durch die $\S \S 80$ Abs. 2 Satz 3 und 28a die Möglichkeit eines gesetzlich abgesicherten, beteiligungsorientierten betrieblichen AGS.
} 
genverantwortung für die eigene Gesundheit sehr weitgehend nachkommt (Becker et al. 2008).

Der Abbau rechtlich verbindlicher AGS-Regelungen ist riskant, denn es besteht die Gefahr, dass an der Ökonomie der kurzen Fristen ausgerichtete Geschäftsstrategien zu einem "gesundheitspolitischen Rollback" in den Betrieben führen (Pickshaus/Urban 2004, S. 222). Beteiligungsorientierte Ansätze als Ausweg brauchen einen langen Atem - eine Herausforderung, die sich durch die Tatsache, dass sich AGS-Investitionen erst mittel- und langfristig auszahlen und zudem schwer zu quantifizieren sind (Becker et al. 2007), ohnehin schon stellt.

Die zweite These geht demnach davon aus, dass das Partizipationsangebot innerhalb des gesetzlich geregelten AGS auch Risiken beinhaltet. Diese äußern sich in der Praxis von Entstandardisierung und Reduzierung von Verbindlichkeit sowie Einschränkung der Reichweite von Arbeitssicherheit und gesundheitsgerechter Arbeitsgestaltung.

Darüber hinaus lassen sich Faktoren benennen, deren Ursachenzuschreibung für einen nur mangelhaft praktizierten AGS zunächst auf die Beschäftigten selbst verweist. Arbeitsschutz kann technisch aufwendig und umständlich erscheinen. $\mathrm{Zu}$ dem wird den Beschäftigten abverlangt, eingeschliffene Arbeitsabläufe und lieb gewonnene Routinen infrage $\mathrm{zu}$ stellen. Gleichwohl steckt z. B. hinter dem Nichtgebrauch bzw. „freiwilligen Verzicht" auf eine Schweißrauch-Absauganlage, wie sie von Managementseite als Beleg für das Eigenverschulden der Beschäftigten an ihrer Gesundheitssituation angeführt wird, auch die Sorge, Zielvorgaben nicht erfüllen zu können. Die durch Kennzahlen und Zielvereinbarungen motivierten Beschäftigten wissen wohl um die Bedeutung ihrer Gesundheit als wichtige Ressource für den Wert der eigenen Arbeitskraft, letzten Endes ordnen sie sie jedoch dem Diktat der Kostensenkung unter, kurz: Das eigene Wohl(ergehen) wird dem des Gesamtunternehmens untergeordnet.

Hinzu kommt: Beschäftigtenbeteiligung führt zu neuen innerbetrieblichen Akteurskonstellationen, die quer zu den etablierten Zuständigkeiten liegen. „Empowerment" der Beschäftigten zur Teilhabe kann dazu führen, dass sich traditionelle Arbeitsschutzakteure wie die Sicherheitsfachkraft plötzlich in einer Konkurrenzsituation wiederfinden. Empirisch lässt sich in vielen Betrieben der Effekt beobachten, dass Fachkräfte für Arbeitssicherheit ihre Arbeit infrage gestellt sehen, wenn die Forderung aufkommt, Gesundheitsschutz ganzheitlich und beteiligungsorientiert zu betreiben. Wird dieses Konkurrenzdenken überwunden und die beteiligungsorientierte Variante als Unterstützung angenommen, bietet die Kooperation von etablierten Experten mit den Beschäftigten jedoch Chancen zu effektiver Problembewältigung und nachhaltigen Gesundheitsschutzmaßnahmen.

Schließlich üben in der betrieblichen Praxis auch tradierte Rollenbilder einen nicht zu unterschätzenden Einfluss aus. So ist es nicht verwunderlich, wenn Betriebsräte berichten, dass die betriebliche Mobilisierung im Gesundheitsschutz traditionell ein schwieriges Unterfangen darstellt (Candeias et al. 2008). Die in jungen und männlich geprägten Belegschaften gepflegten Ideale von Stärke und Unversehrtheit machen es gesundheitsbewussten Verhaltensweisen schwer, Fuß zu fassen.

\subsection{BEFÄHIGUNG ZUR BETEILIGUNG}

Wir haben es letztendlich mit zwei unterschiedlich wirksamen Aktivierungsstrategien zu tun: Eine an ökonomischen Zielvorgaben orientierte Logik soll die Aktivierung in Richtung höherer Produktivität, Effektivität und Selbstausbeutung anstoßen. Eine Aktivierung zu eigenverantwortlicher AGS-Gestaltung zielt zum Teil in die entgegengesetzte Richtung. Allein, dass Beschäftigtenbeteiligung während der Arbeitszeit organisiert werden muss, mindert zunächst die produktiv-aktiven Zeiten von ganzen Gruppen oder Abteilungen. Wenn diese dann noch Maßnahmen beschließen, die die Fehlbelastungen reduzieren, dann können sich große Diskrepanzen zu den ursprünglichen Zielvorgaben eröffnen. Um diesem Beteiligungsrisiko oder -dilemma zu entgehen, ist die Schaffung von Freiräumen notwendig, die Überlegungen zu einer beteiligungsorientierten Verbesserung des AGS überhaupt erlauben. Dazu bedarf es sowohl einer Vermittlung von AGS- als auch von Beteiligungskompetenz, wobei hier der Fokus auf die Befähigung zu Beteiligung gelegt werden soll.

Wenn sich die Qualität von AGS unter anderem daran bestimmt, wie stark Beschäftigte einbezogen werden und sich selbst aktiv beteiligen, müssen begünstigende Faktoren vor allem darin zu suchen sein, wie sehr sie diesen Ansatz befördern. Einen herausragenden Stellenwert nimmt hier die Kopplung an gesetzlich abgesicherte institutionelle Formen der Interessenvertretung ein, die am ehesten das Potenzial in sich bergen, durch die Einlösung des Beteiligungsanspruchs auch dem Gesundheitsmanagement zu nachhaltiger Wirksamkeit zu verhelfen. Als dritte These lässt sich also formulieren: Die Chancen eines positiv aktivierenden AGS setzen eine gesetzlich abgesicherte Befähigung zur Beteiligung voraus.

Beschäftigte können einen profunden Beitrag zur Analyse von Gefährdungen, Belastungen und Bewältigungsressourcen leisten. Beteiligungsorientierter Gesundheitsschutz kann dazu beitragen, dass Beschäftigte die Erfahrung machen, erfolgreich die Arbeitssituation beeinflussen und letztlich verbessern zu können und somit die Arbeitsanforderungen als gestaltbar erleben. Vor dem Hintergrund alternder Belegschaften und eines erweiterten Aufgabenspektrums für deren gewählte Vertretungen erweisen sich beteiligungsorientierte Ansätze im Bereich des „Gesundheitsmanagements" als erfolgreiches Empowerment. Die eingeschränkte Wirksamkeit des betrieblichen AGS - so der Umkehrschluss - könnte an der Experten- und Top-DownPerspektive liegen, wie sie in vielen Betrieben noch vorherrscht und in daran orientierten wenig nachhaltigen Arbeitsschutzpraxen mündet.

\subsection{BETEILIGUNGSVORAUSSETZUNG: ERKÄMPFTE FREIRÄUME}

Die angeführten Argumente - im Sinne eines positiv gewendeten Aktivierungsbegriffs - verdeutlichen, dass die erweiterten Beteiligungsmöglichkeiten im AGS auf verschiedene strukturelle Erschwernisse und betriebspolitische Hürden treffen, die es zu überwinden gilt. Die vierte These dieses Beitrags lautet daher: Positive Aktivierung zur Beteiligung im AGS ist voraussetzungsvoll und bedarf eines Freiraums, der gegebenenfalls erkämpft werden muss.

Dies erfordert spezifische Ressourcen, die von machtvollen Akteuren im mikropolitischen „Spiel“ (Crozier/Friedberg) eingebracht werden. Die Schaffung von Freiraum soll sicherstellen, dass der Einfluss anderer Funktionslogiken (wie jener des Marktes oder der Hierarchie) innerhalb dieses Raums geschwächt wird. In diesen Freiräumen müssen die Akteure über so 
viel Deutungshoheit und Handlungsautonomie verfügen, dass sich Investitionen in AGS nicht allein an ökonomischen Imperativen messen lassen müssen oder an hierarchischen Unterstellungsverhältnissen und Weisungsbefugnissen scheitern. Vielfach sind es auch unternehmenskulturell verankerte Leitbilder und Ideologien, die dazu beitragen, dass Marktgrenzen sukzessive in die Unternehmen hineinverlagert werden (Brinkmann et al. 2008) und AGSProjekte erschweren (Becker et al. 2007).

Das Ausmaß des Freiraums hängt von verschiedenen Faktoren ab und kann durch unterschiedliche Strategien beeinflusst werden. Letztlich geht es darum, Macht zu akkumulieren und, mithilfe akkumulierter Macht, Interessen zur Durchsetzung zu verhelfen. Dies kann gelingen, indem man den eigenen Anliegen in politischen Prozessen Gehör verschafft und Nachdruck verleiht, aber auch dadurch, dass eigene Verhaltensweisen unvorhersehbar gehalten werden, um so die Handlungsmöglichkeiten und Freiräume der Gegenseite einzuschränken. Die Kontrolle über Handlungsräume bietet Organisationsmitgliedern die Möglichkeit, Situationen kreativ und taktisch zum eigenen Vorteil zu nutzen und zugleich eigene Freiräume vor Restriktionen zu schützen.

\section{Betriebliche Gesundheits- politik - eine Fallstudie}

Der folgende Betriebsfall eines deutschen Automobilherstellers soll hier beispielhaft illustrieren, welche Strategien Betriebsratsakteure zur Schaffung von Freiraum im betrieblichen AGS entwickeln und zum Einsatz bringen können und mit welchen Herausforderungen sie dabei konfrontiert sind. Der konkrete betriebliche Kontext: Beide Betriebsparteien, Management und Betriebsrat, verfolgen verschiedene Konzepte, wie mit der gesetzlichen Vorgabe der Durchführung einer Gefährdungsbeurteilung der Arbeitsplätze umzugehen ist. Obwohl der Prozess noch nicht endgültig abgeschlossen ist - das heißt, ein gemeinsamer Beschluss darüber steht noch aus -, lohnt sich die Analyse dieses Betriebsfalls, weil deutlich wird, welche Voraussetzungen für die Entfaltung einer auf den Einzelnen abzielenden Aktivierungslogik überhaupt gegeben sind.

\subsection{VON DER MACHTLOSIGKEIT ...}

Obwohl das Arbeitsschutzgesetz bereits 1996 die Gefährdungsbeurteilung ins Zentrum des betrieblichen AGS-Geschehens rückte, gab es im betreffenden Unternehmen sowohl von Betriebsrats- als auch von Managementseite lange keine Initiativen, dieser gesetzlichen Pflicht nachzukommen. Die Initialzündung für die Bearbeitung dieses Themas bildete die Rechtssprechung des Bundesarbeitsgerichts (BAG) 2004, die die Mitbestimmungsrechte von Betriebsräten an der Gefährdungsbeurteilung (GB) bestätigt (Gäbert/Maschmann-Schulz 2008). Im Zuge dessen wurde das Betriebsratsgremium erstmals auf dieses Instrument und die damit verbundenen Gestaltungsmöglichkeiten im AGS aufmerksam. Mit der GB, so erkannte man im Zuge des BAG-Urteils, bekommt der Betriebsrat (BR) durch die gesetzliche Absicherung ein Instrument an die Hand, mit dem er sich den nötigen Freiraum schaffen kann, um arbeitspolitische Felder erschließen und bearbeiten zu können, auf die er vormals keinen Zugriff hatte. Eine Anfrage des BRGremiums an die Geschäftsführung, ob die Umsetzung einer GB geplant sei, blieb zunächst unbeantwortet.

Der betriebliche AGS wird vom BR als Gestaltungsfeld in dieser Zeit eher vernachlässigt, was ein BR-Mitglied rückblickend damit begründet, dass im Zuge einer langen Restrukturierungsphase des Unternehmens in den 1990er Jahren zunächst „einmal andere Themen auf der Agenda standen.“ Damit einher geht eine faktische Machtlosigkeit des BR auf diesem betriebspolitisch umkämpften Feld.

Welche Probleme die Vernachlässigung eines funktionierenden Gesundheitsmanagements nach sich ziehen kann, führten schließlich die jährlichen Gesundheitsberichte der Betriebskrankenkasse vor Augen. Sie dokumentierten, dass die Arbeitsausfalltage seit Mitte der 1990er Jahre signifikant anstiegen. Anhand dieser Ergebnisse ließ sich auf Versäumnisse im Bereich der Gesundheitsprävention schließen. Die Berichte der Betriebskrankenkasse sorgten schließlich dafür, dass der Zustand von Arbeitssicherheit und Gesundheitsschutz in das Blickfeld der gesamten Belegschaft rückte. „Die Statistiken“, so schildert es ein BR-Mitglied, „waren insofern nützlich, als sie bei der Belegschaft einen Aha-Effekt auslösten: ,Hier muss etwas passieren'. Einige traten an uns heran und forderten von uns, für sie, für ihre Arbeitsbedingungen etwas zu tun". Die auf diese Weise aktivierten Beschäftigten erwiesen sich für den BR als wichtige Ressource, um Unterstützung zu mobilisieren und in den folgenden Konflikten mit dem Management Macht aufzubauen.

Während sich der BR in der Folgezeit der Analyse und Bekämpfung der Ursachen gestiegener Belastungen verschrieb, reagierte die Geschäftsführung auf den massiven Anstieg der Krankheitsrate mit einem repressiven Disziplinierungskurs, den sie argumentativ dadurch abzustützen versuchte, dass sie eine „Drückebergerdebatte“ entfachte: „Das sind die Leute, die das Unternehmen auf schäbigste Art und Weise ausgenutzt haben. Die Krankmacher hier schaden dem Unternehmen und damit jedem Einzelnen“, so der Personalchef, öffentlich bei einer Betriebsversammlung. Bei diesem Vorgehen des Managements geht es nicht mehr nur darum, die Belegschaft durch Appelle zu aktivieren. Beschäftigte, die mit den Anforderungen nicht Schritt halten können - dies zeigt das Zitat - werden diffamiert, indem ihnen eine intentionale Schädigung des Gesamtwohls unterstellt wird.

Innerhalb des Konzerns war der untersuchte Standort unter Druck geraten, weil er im Vergleich zu den anderen Betrieben überdurchschnittlich hohe Fehlzeiten aufwies - zurückzuführen allerdings auch auf die konzerninterne Spitzenstellung bei den produktivitätsausweisenden Kennziffern. Als eine der ersten betrieblichen Maßnahmen wurde ein gestuftes Personalgesprächsprogramm aufgelegt. „Wir vom Betriebsrat haben ein sogenanntes Anwesenheitsprogramm geduldet, was Fehlzeitengespräche beinhaltet", gibt ein BR-Mitglied selbstkritisch zu Protokoll.

Eine weitere betriebliche Disziplinierungsmaßnahme bestand in der Identifizierung von Beschäftigten mit über 30 Ausfalltagen im Jahr. „Auch wurden Beschäftigte ins Visier genommen, die den schlechtesten Stand bei den Kollegen haben" (Betriebsratmitglied). Bald schon wurde gegen zehn dieser - wie es der Personalchef ausdrückte - „Sozialschmarotzer“ krankheitsbedingte Kündigungen ausgesprochen. Ein Teil der Beschäftigten ließ sich für diese nicht gesetzeskonforme Kündigung finanziell entschädigen, andere fochten die Entscheidung gerichtlich an. Die Kündigung eines besonders benachteiligten Beschäftigten nutzte der BR dazu, das 
Vorgehen des Managements zu skandalisieren. Die Motivation zu diesem mikropolitisch geschickten Vorgehen speist sich aus einem tiefen Ungerechtigkeitsempfinden des Betriebsrates: „An dem Fall ist mir erst mal klar geworden: Nicht die Leute sind es, die das Unternehmen ausnutzen, sondern umgekehrt. Die Leute rackern für das Unternehmen und werden dann einfach fallen gelassen. Sowas muss man verhindern. Dagegen muss man etwas tun."

Diese Erfahrung, so schildert es die Interviewpartnerin, gab den entscheidenden Impuls, sich „aktiv als Interessenvertreterin zu engagieren " - ein Beispiel für eine gesetzlich abgesicherte, machtvolle positive Aktivierung, die darin besteht, sich in einem vormals vom Management besetzten betriebspolitischen Feld einbringen und damit die Arbeitsbedingungen im Sinne der Beschäftigten mitgestalten zu können.

\section{2 ... ZUR GEWINNUNG MACHTPOLITISCHEN FREIRAUMS}

Ein Teil des Betriebsrats versuchte machtpolitischen Freiraum zurückzugewinnen und setzte sich für die Weiterbeschäftigung des Kollegen ein. Dafür legte man dem Arbeitsgericht die formalen Fehler des Managements dar, die eine Kündigung des Beschäftigten nicht rechtfertigten. Das Außerkraftsetzen hierarchischer Ordnungsprinzipien gelang in diesem Fall auch deshalb, weil die Anrufung des Arbeitsgerichts die Nichteinhaltung gesetzlicher Vorgaben durch die Geschäftsführung - nämlich die fehlende GB - offenbarte. Die Erkenntnis, dass das Instrument GB eine bislang noch unterschätzte Ressource für die Durchsetzung von Belegschaftsinteressen ist, gab der Interessenvertretung zusätzlichen Aufwind, dieses Thema engagiert zu bearbeiten.

In der Folge formierte sich eine Gruppe von Betriebsräten, die sich dem Ziel verschrieb, eine Gefährdungsbeurteilung durchzuführen, und eine darauf ausgerichtete Strategie entwickelte. Das Vorgehen spielte sich auf vier Ebenen ab, die hier im Detail und in ihrer betrieblichen, zum Teil sich überschneidenden Wirksamkeit nachgezeichnet werden sollen:

- Erwerb von Analysekompetenz,

- Gewinn von innerbetrieblicher Diskurshoheit,

- Beteiligung von Beschäftigten,

- Schaffung und Verstetigung strategischer Handlungsfähigkeit.
ERWERB VON ANALYSEKOMPETENZ

Zur kompetenten Bearbeitung von AGSProblemen in Unternehmen bedarf es einer umfangreichen Kenntnis des zugrundeliegenden arbeitstechnischen, -organisatorischen,-medizinischen und -rechtlichen Wissens. Um als Interessenvertreter auf Augenhöhe mit Sicherheitsfachkraft und Geschäftsführung über Beteiligung im AGS zu verhandeln, muss sich der Betriebsrat mit dieser Materie vertraut machen, sich also zum Experten auf diesen Gebieten entwickeln.

Im hier behandelten Betriebsfall wird ein hoher Aufwand betrieben, diese Voraussetzung zu erfüllen: Der Leiter des Betriebsratsausschusses für Arbeitsschutz besuchte eine Vielzahl von Seminaren, die ganz spezifische, auf seine Interessenvertretungsfunktion zugeschnittene Fragen beantworteten. So verfügt der Betreffende z. B. über Kenntnisse der Mitbestimmungsrechte im Prozess der Gefährdungsbeurteilung oder der Erzwingung einer GB über die Einberufung einer Einigungsstelle. Diese Kompetenzen führen zu einer Neujustierung der innerbetrieblichen Machtkonstellation zugunsten des Betriebsrats, denn er hat mittlerweile umfangreichere Kenntnisse bezüglich der neuen Anforderungen an eine GB als die eher mit klassischen Gefährdungen und Ansätzen vertraute Arbeitssicherheitsabteilung des Unternehmens. Ein Betriebsratsmitglied dazu: „Der Personalchef weiß mittlerweile, dass er mich bei der Gefährdungsbeurteilung braucht. Ich kenne mich da mittlerweile viel besser aus. Da lass ich mir auch von keinem hier mehr was vormachen, ganz im Gegenteil, die stecke ich bei diesem Thema in die Tasche".

\section{GEWINN VON DISKURSHOHEIT}

Das Vorgehen des Betriebsrats macht deutlich, dass es nicht nur um die Aneignung von Kompetenz auf diesem Gebiet geht, sondern dass vielmehr Wissensvorsprünge erarbeitet werden, die die Voraussetzung dafür sind, innerbetrieblich die Diskurshoheit auf diesem Gebiet zu erkämpfen. In den Worten eines Betriebsrats-Mitglieds: „Man muss sich als Betriebsrat einfach verschiedene Kompetenzen erarbeiten, um der anderen Seite auf Augenhöhe begegnen zu können. Sonst wird man niedergemacht." Erfolgreiche oder „positive“ Aktivierung - im Sinne des Besetzens betriebs- politischer Felder zur Verbesserung der (Arbeits-)Bedingungen entsprechend der Vorstellungen von Beschäftigten - ist, wie das Zitat zeigt, kein Selbstläufer. Vielmehr müssen beteiligungsmotivierte Akteure auf spezifische Voraussetzungen aufbauen können, die hier als Strategien des Freiraumschaffens beschrieben werden.

Dabei achten die BR-Akteure darauf, dass die Schulungsmaßnahmen von einschlägigen Arbeitswissenschaftlern angeboten werden, die auch in betriebspolitisch brisanten Fragen viel Erfahrung haben. Entsprechende rechtliche Grundlagen wurden z. B. in mehreren Seminaren des Richters am Bundesarbeitsgericht vermittelt, der 2004 das Urteil durchgesetzt hatte. Im späteren Verlauf der GB-Vorbereitung wurde wissenschaftliche Expertise einer universitären Forschungsgruppe hinzugezogen, die bereits in anderen Unternehmen verschiedene Verfahren erfolgreich begleitet hatte. Kontrastierend sei hier kurz erwähnt, dass die Geschäftsleitung die Auswahl ihrer externen Experten zur Durchführung einer GB in erster Linie anhand der zu erwartenden Kosten vornahm.

Neben dem qualitativen Wissensvorsprung für die Beschäftigtenvertretung gegenüber den betrieblichen Experten und Verantwortlichen geht es auch um die Verbreitung der erworbenen Kompetenzen. Interne Betriebsrats- und Ausschusssitzungen wurden auch dazu genutzt, die Seminarerfahrungen weiterzugeben.

Wie Crozier/Friedberg herausgearbeitet haben, darf Macht nicht Strukturen als solchen zugeschrieben werden. Vielmehr kommt es darauf an, dass Akteure sie anderen gegenüber wirksam zur Geltung bringen. Macht resultiert demzufolge aus dem Vermögen, in Interaktionen relevante Handlungsbedingungen des anderen zu kontrollieren (Crozier/Friedberg 1994/ 1979). Als besonders nachhaltige, Freiraum schaffende Strategie erwies sich ein ganztägiges Betriebsratsseminar, das in einem einstimmigen Betriebratsbeschluss zur Durchführung einer GB mündete. Als die Zustimmung der eher Co-Managementorientierten BR-Fraktion als Reaktion auf die Blockadehaltung der Geschäftsführung bröckelte, konnte sich der andere BR-Teil auf diesen Beschluss berufen. Unternehmenskulturell begründete Blockadehaltungen innerhalb des Interessenvertretungsgremiums, die dem Management bereits häufig in die Hände spielten, konnten aufgebrochen werden. Der Betriebsratsvor- 
sitzende - obwohl selbst auch eher CoManagement-orientiert und entsprechend dysfunktional hinsichtlich der Durchsetzungschancen einer GB argumentierend bestätigte noch mal den verbindlichen Charakter dieses Beschlusses: „Dahinter können wir jetzt nicht mehr zurück.“

\section{BETEILIGUNG VON BESCHÄFTIGTEN}

Zugleich wurden mit diesem Beschluss die Weichen für die Schaffung innerbetrieblicher Diskurshoheit gestellt. Der BR machte die Gefährdungsbeurteilung zu seiner Sache und kündigte deren Durchführung in Informationsmaterialien und auf der Betriebsversammlung an. Als die Personalleitung eine Entscheidung über einen gemeinsamen Fragebogen zur Erfassung von gesundheitlichen Belastungen blockierte, entschied sich der Betriebsrat dafür, ein eigenes, der GB vorgeschaltetes Projekt in Form einer aktivierenden Befragung durchzuführen. Das Potenzial dieser Aktivierungsstrategie besteht darin, dass die Beschäftigten durch die Befragung über bestehende Probleme informiert, dafür sensibilisiert und für deren Bekämpfung mobilisiert werden. Aktivierungsstrategien in Form einer Befragung sind immer auch Beteiligungsstrategien. In diesem Fall ging es darum, ein Meinungsbild der Belegschaft einzuholen. Dazu wurden in allen Abteilungen Fragebögen ausgegeben und erläutert. Auch der anonymisierte Rücklauf sowie die Weiterverarbeitung der Daten, die an externe Experten vergeben wurden, war sichergestellt. Weitere Beteiligungsschritte waren im Anschluss an die Befragung durch die Möglichkeit der Ergebnisdiskussion vorgesehen sowie im Prozess der anstehenden Gefährdungsbeurteilung.

\section{STRATEGISCHE HANDLUNGS- FÄHIGKEIT}

Die Befragung ermöglicht auch, Belastungsschwerpunkte im Unternehmen aufzudecken. Sind die Probleme durch die Befragung der Beschäftigten erst einmal benannt, kann der BR Handlungsdruck aufbauen und die Geschäftsführung in Zugzwang bringen. Mithilfe der Befra- gungsergebnisse, so das Kalkül des BR, soll die Notwendigkeit einer GB allen im Betrieb vor Augen geführt werden. Damit schafft sich der BR bei der Belegschaft zusätzliche Legitimation für sein Vorgehen.

Obwohl es mehrere Ansätze vonseiten des Managements gibt, das Ziel des Betriebsrates - die Durchführung einer Gefährdungsbeurteilung - zu unterlaufen, zeigt sich, dass die Interessenvertretung ihre strategische Handlungsfähigkeit sukzessive ausbaut und sich darüber autonomieund machterweiternde Freiräume schafft. Neben den bereits beschriebenen strategischen Handlungsfeldern (Kompetenzerwerb, Diskurshoheit, Beteiligung) erfordert das Ziel des Betriebsrats eine mikropolitisch geschickte Vorbereitung von Entscheidungen und die Suche nach externen Bündnispartnern. Nicht zuletzt durch die Seminare bestehen zahlreiche Kontakte zu Arbeitsrechtlern und Gewerkschaftern auf Landes- wie Bundesebene. Das Gutachten eines Juristen, das die Vorgehensweise des Betriebsrates bei der Anbahnung der GB bestätigte, und die offene Ankündigung, notfalls auch den Weg über die Einigungsstelle zu gehen, änderten auch die Haltung der GL: „Der Umgang hat sich nun gedreht. Von der schroffen Ablehnung zu: ,Na ja, wir können doch mal über alles reden“" (BR-Mitglied).

\section{$\angle$ \\ Schlussfolgerung und Ausblick}

Das Fallbeispiel zeigt, dass der erkämpfte Freiraum einen faktischen Machtgewinn der Interessenvertretung mit sich bringt. Sowohl das Arbeitsschutzgesetz als auch das Betriebsverfassungsgesetz bieten potenziell geschützte Freiräume. Es ist an den Interessenvertretungen, diese zur Durchsetzung von Belegschaftsinteressen zu nutzen bzw. auszuweiten. Es ist durchaus denkbar, dass anstelle einer gewählten Vertretung eine kurzzeitig sich zusammenfindende Gruppe von Beschäftigten ihre Interessen bündelt und sich zu einem strategischen Vorgehen entschließt, um Gestal- tungsfreiräume zu schaffen. Allerdings: Die Voraussetzungen dafür sind für in der Betriebsratsarbeit erfahrene Belegschaftsgremien weitaus günstiger. Allein durch die regelmäßige Kommunikation untereinander und die rechtlich garantierte Freistellung vom Arbeitsprozess sowie die Information der Beschäftigten und andere Routinen haben gewählte Mitbestimmungsorgane klare Vorteile - ja, sie sind geradezu Voraussetzung, um erfolgreich dem Anspruch des neuen AGS als eigenverantwortlich zu regelnde Angelegenheit der Beschäftigten gerecht werden zu können.

Bei einer schleichenden Erosion der Arbeitssicherheits- und Gesundheitsschutzstandards unter den Bedingungen des Finanzmarktkapitalismus werden primär diejenigen Beschäftigten das Potenzial der eigenverantwortlichen, gesundheitsgerechten Gestaltung von Arbeitsbedingungen umfangreich nutzen können, die auf funktionierende interessenpolitische Vertretungsstrukturen mit Offenheit für Beteiligung und auf partizipationsorientierte Unternehmenskulturen zurückgreifen können. Die Auseinandersetzung mit dem Fallbeispiel führt vor Augen, dass es dabei darauf ankommt, bestehende Freiräume zu nutzen und neue Freiräume zu erkämpfen.

Der Betriebsfall gibt zugleich Hinweise auf einen weiteren interessanten Zusammenhang: Während sich in der empirischen Praxis eher Beispiele dafür finden lassen, dass im Fall einer Unternehmenskrise Beschäftigte ihre Gesundheit zugunsten des Arbeitsplatzerhalts hintenanstellen, führt im hier betrachteten Unternehmen eine drohende Werksschließung dazu, dass Beschäftigte die Bedeutung der Gesundheitserhaltung als Voraussetzung für die Reproduktion ihrer Arbeitskraft reflektierten. Unter dem Damoklesschwert eines möglichen Arbeitsplatzverlustes kommen sie zu dem Schluss, dass sie nur dann eine Chance auf eine neue Anstellung haben, wenn sie eine Selbstbewirtschaftung betreiben, die auf den nachhaltigen Erhalt ihrer Gesundheit ausgerichtet ist. Dies kann, wie in diesem Beispiel, den Impuls für eine positive Aktivierung geben. 


\section{LITERATUR}

Becker, K./Brinkmann, U./Dörre, K./Engel, T. (2007): Die Kosten des Sparens am Arbeits- und Gesundheitsschutz, in: Engel, K. H./Krauß, M. (Hrsg.): Betriebsleiter. Praxishandbuch IV/2007 - Kissing, S. 12.3.0112.3.21

Becker, K./Brinkmann, U./Engel, T. (2007): Die Haut auf dem Markte. Betrieblicher Gesundheitsschutz im Marktkapitalismus, in: Prokla 3,

S. 383-401

Becker, K./Brinkmann, U./Engel, T. (2008): Beschäftigte als „Unternehmer der eigenen Gesundheit“?, in: Bogedan, C./Müller-Schoell, T./Ziegler, A. (Hrsg.): Demografischer Wandel als Chance, Hamburg, S. 173-191 Brinkmann, U./Benthin, R./Dörre, K. (2008): Culture Club oder demokratische Teilhabe? Unternehmenskultur und Mitbestimmung im neuen Marktkapitalismus, in: Benthin, R./Brinkmann, U. (Hrsg.): Unternehmenskultur und Mitbestimmung, Frankfurt a. M., S. 23-72

Candeias, M./Röttger, B./Brinkmann, U./Dörre, K./Schulz, M. (2008): Betriebsräte in der Zivilgesellschaft, unv. Forschungsbericht für die HansBöckler- und die Otto-Brenner-Stiftung, Jena

Crozier, M./Friedberg, E. (1979/1994): Macht und Organisation. Die Zwänge kollektiven Handelns, Königstein

Dörre, K. (2002): Kampf um Beteiligung. Arbeit, Partizipation und Industrielle Beziehungen im flexiblen Kapitalismus, Wiesbaden

Fuchs, T. (2007): Wie nachhaltig ist die Arbeitswelt in Deutschland?

Arbeit im Kontext von Einkommen, Sicherheit und Arbeitsqualität, Stadtbergen
Gäbert, J./Maschmann-Schulz, B. (2008): Mitbestimmung im Gesundheitsschutz. Handlungshilfe für Betriebsräte, Frankfurt a. M. Lessenich, S. (2008): Die Neuerfindung des Sozialen: Der Sozialstaat im flexiblen Kapitalismus, Bielefeld

Morschhäuser, M. (2003): Gesund bis zur Rente? Ansatzpunkte einer alternsgerechten Arbeits- und Personalpolitik, in: Badura, B./Schellschmidt, H./Vetter, C. (Hrsg.): Fehlzeiten-Report 2002, Berlin, S. 59-71 Opielka, M. (2008): Sozialpolitik. Grundlagen und vergleichende Perspektiven, Reinbek

Pickshaus, K. (2007): Was ist gute Arbeit?, IG Metall Projekt Gute Arbeit (Hrsg.): Handbuch "Gute Arbeit" , Hamburg, S. 16-31

Pickshaus, K./Urban, H. J. (2004): "Gute Arbeit“ - Eine arbeits- und gesundheitspolitische Initiative der IG Metall, in: Arbeit 3, S. 220-228 Reusch, J. (2009): Gute Arbeit unter Druck: Deregulierung und Ökonomisierung, in: Schröder, L./Urban, H. J. (Hrsg.): Gute Arbeit, Frankfurt a. M., S. 169-182

Sauer, D. (2009): Gute Arbeit statt humaner Arbeit - mehr als ein Wechsel des Begriffs?, in: Schröder, L./Urban, H. J. (Hrsg.): Gute Arbeit, Frankfurt a. M., S. 135-145

Schröder, G. (2000): Die zivile Bürgergesellschaft. Anregungen zu einer Neubestimmung der Aufgaben von Staat und Gesellschaft, in: Neue Gesellschaft/Frankfurter Hefte 4, S. 200-207 